\title{
A Partial Equilibrium Theory for Drops and Capillary liquids
}

\author{
1,2 Alan W. Searcy*, 2 Dario T. Beruto, and ${ }^{2}$ Fabrizio Barberis \\ ${ }^{1}$ Lawrence Berkeley National Laboratory and Department of Materials Science and \\ Engineering, University of California, Berkeley, CA 94720, USA, \\ ${ }^{2}$ Department of Engineering Materials and Building Science, Faculty of Engineering, \\ University of Genoa, 16129, Italy *To whom correspondence should be addressed. E- \\ mail: asearcy@,berkeley.edu
}

\begin{abstract}
The two-century old theory of Young and Laplace retains a powerful influence on surface and interface studies because it quantitatively predicts the height of rise of capillary liquids from the contact angles of drops. But the classical theory does not acknowledge that equilibrium requires separate minimization of partial free energies of one-component liquids bonded to immiscible solids. We generalize a theorem of Gibbs and Curie to obtain a partial equilibrium (PE) theory that does so and that also predicts the height of capillary rise from contact angles of drops. Published observations and our own measurements of contact angles of water bonded to glass and Teflon surfaces support the conclusion of PE theory that contact angles of meniscuses and of drops are different dependent variables. PE theory provides thermodynamic and kinetic guidance to nanoscale processes that the classical theory obscures, as illustrated by examples in our concluding section.
\end{abstract}

\section{Equations of PE theory}

The partial Gibbs energy, $\mathrm{G}_{\mathrm{p}}$, of a particle of $n$ interfaces, each of which can bond to a solid, is in a local thermodynamic equilibrium state when

$$
\mathrm{dG}_{\mathrm{p}}=\mathrm{d} \Sigma_{n}\left(\gamma+\lambda_{\mathrm{i}}\right) \mathrm{Ai}_{\mathrm{i}}>0
$$

for any differential change in shape at constant mass and temperature, where $\lambda_{i}$ is zero at unshared interfaces. The summation is over the particle interfaces, and $\mathrm{A}_{i}$, for example, is the area of interface i. For non-bonded particles, Eq. 1 reduces to the Gibbs-Curie theorem. For small drops that bond to only one planar surface Eq. 1 is satisfied when

$$
\gamma \cos \theta \mathrm{d} a=-\left(\gamma+\lambda_{a}\right)
$$

where $\theta_{\mathrm{d} a}$ is the contact angle of the drop on surface $a$, and $\left(\gamma+\lambda_{a}\right)=\psi_{a}$ is the net partial free energy of forming a unit area of drop surface from the bulk liquid and bonding it to solid $a$.

- In a capillary, a liquid is attracted to two surface instead of one and - $2 \psi a / \gamma=\cos \theta \mathrm{m} a$. Therefore, $\theta \mathrm{m} a$ is predicted to be zero as long as $\theta \mathrm{d} a$ is less than $60^{\circ}$. The height of rise of the capillary liquids with zero contact angles is described by

$$
\Delta \rho g(h \mathrm{~b}+\mathrm{r} / 3)=-2 \psi_{a} / \mathrm{r} \text {. }
$$

But - $\psi_{a}=\gamma$. Therefore, for liquids that have hemispherical capillaries, the prediction of PE theory from contact angles of drops reduces to the Laplace equation. 


\section{Experimental Tests}

If PE theory is correct, $\theta_{\mathrm{m}}=0$ for all liquid-solid systems for which $\theta_{\mathrm{d}}$ is no more than $60^{\circ}$. We confirm this prediction for the only case for which both $\theta_{\mathrm{d}}$ and $\theta_{\mathrm{m}}$ have been measured, water on glass and between glass surfaces. The classical assumption that $\theta_{\mathrm{m}}=$ $\theta_{\mathrm{d}}$ correctly predicts the height of rise because that assumption is equivalent to accepting Eqs. 2 and 3.

Classical theory predicts that the profile of water between parallel surfaces as dissimilar as glass and Teflon must have an inflection point pinned as shown in Fig. 2

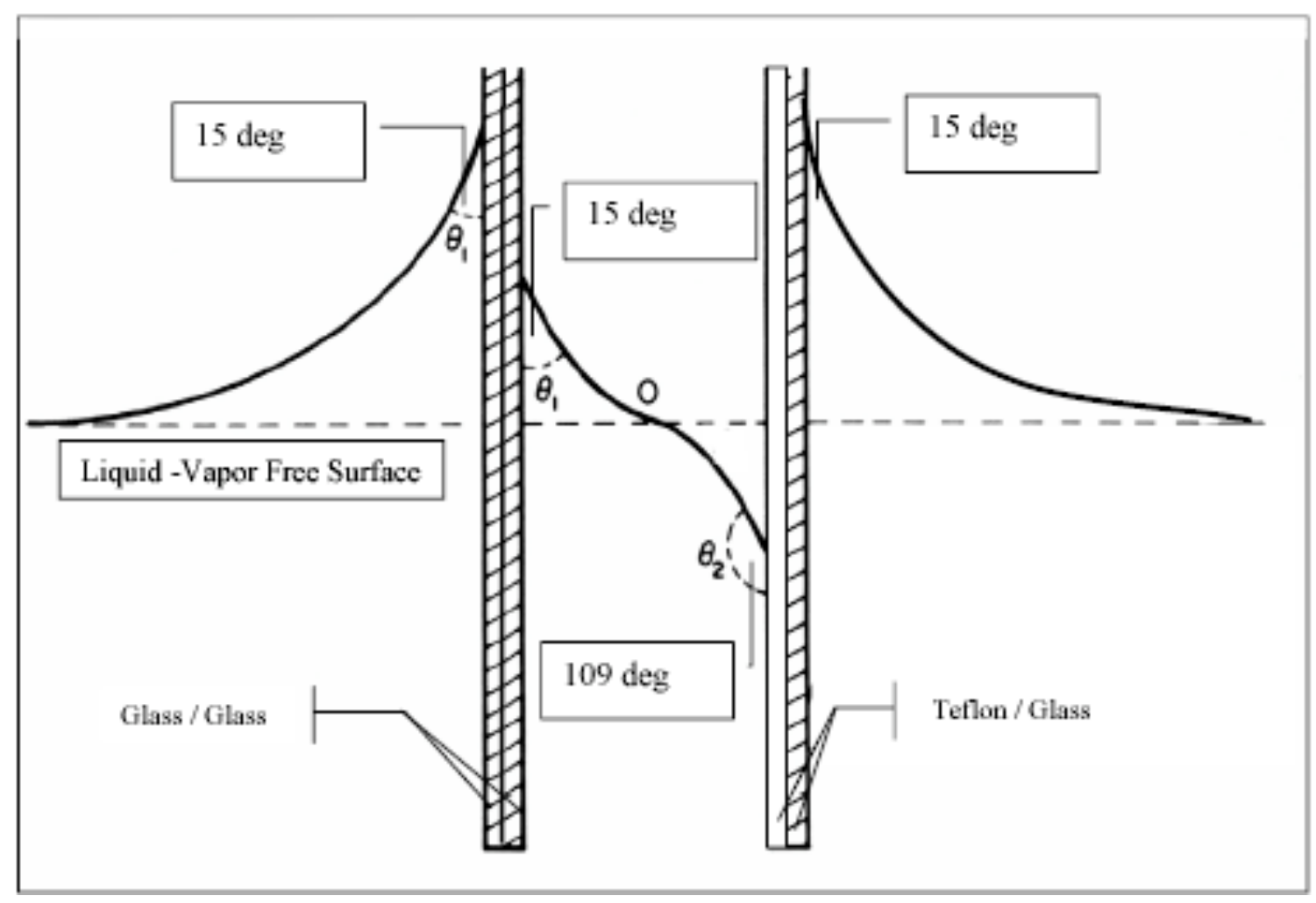

Figure 2

Modification of Bikerman's schematic drawing (29) of the profile predicted by the Young-Laplace theory for a liquid between surfaces on which one contact angle is less than $90^{\circ}$ and the other is greater. In our experiments, the Teflon was backed by a glass plate, as shown. The meniscus angles and the inflection point at the level of the reservoir surface are predicted by the Young-Laplace theory from angles measured for water drops on glass and Teflon surfaces.

Fig. 3 shows three contradictions of the classical predictions of Fig. 2: There is capillary rise, which in ref 19 is shown to be $\sim 10$ times as great as we found in Fig. 3 when the spacing between surfaces is reduced to $0.1 \mathrm{~mm}$. The contact angle of the inner water meniscus is $(40 \pm 2)^{\mathrm{O}}$, about $25^{\circ}$ greater than measured on the outer glass surface. The angle formed by the meniscus at the Teflon surface is $(101 \pm 2)^{\circ}$, which is about $8^{\circ}$ less than that of a drop on Teflon. And the meniscus curvature at the Teflon surface is 
concave rather than convex.

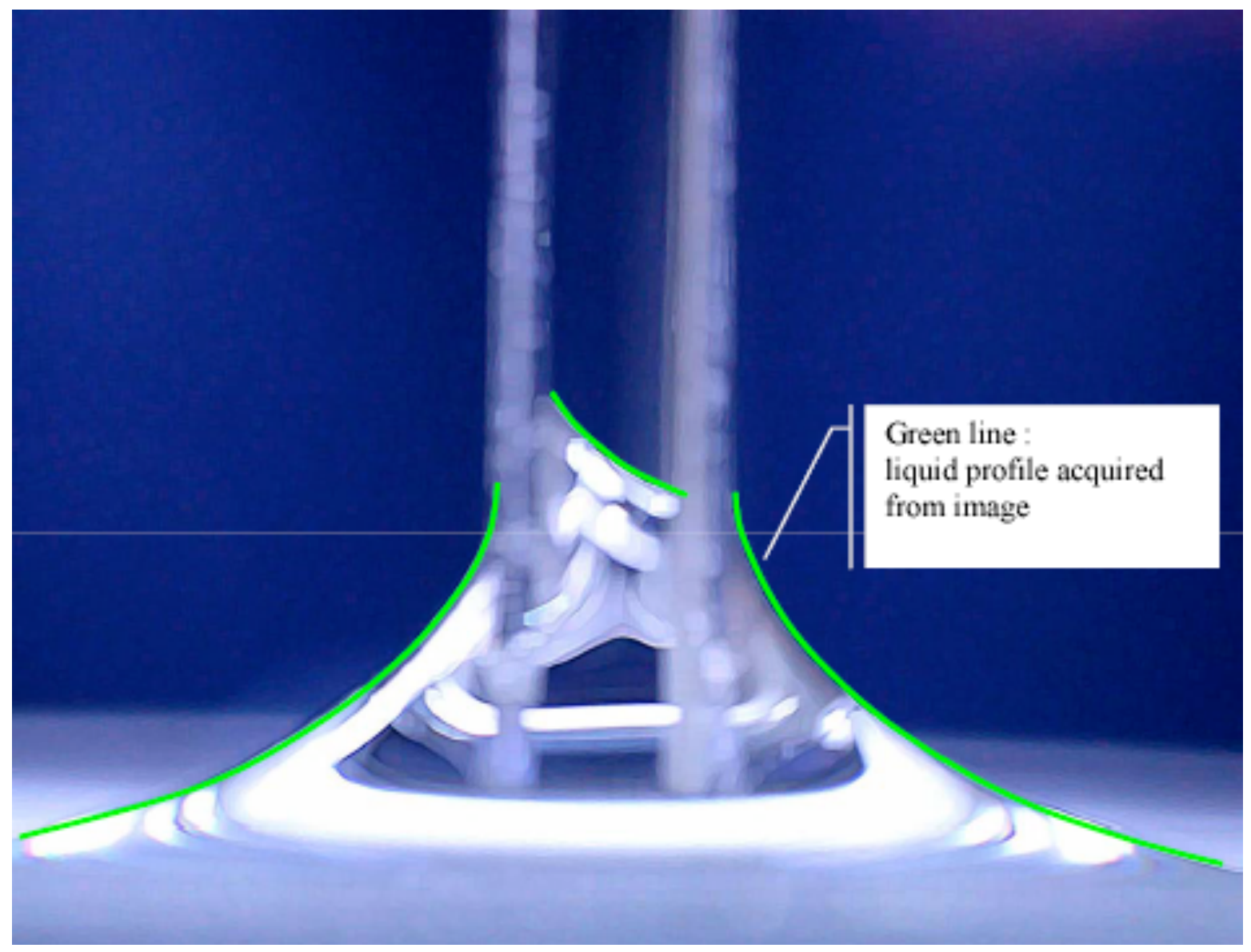

Figure. 3

Photographed profile of water between glass and Teflon surfaces. Contact angles of the water on the outer surfaces, which are far from reservoir walls, are the same as for water drops on glass. But the observations contradict three predictions of classical theory illustrated in Fig. 2. Capillary rise, a change in contact angles from those of drops, and an uninflected, but asymmetric, meniscus profile is predicted by the partial equilibrium theory of this paper.

\section{Three of five Applications of PE theory cited in the concluding section}

In 1974 Cahn and Hoffman (27) used a sophisticated vector formalism to extend the Wulff construction for equilibrium shapes of isolated particles to particles on surfaces by assuming that the center that the particle would have if it were completed with the same cross-sections can be identified as $\pm \Delta \gamma_{l v}$, the unit free energy of particle-vapor interface free energy. Because Wulff particles are a subset of particles for which the Gibbs-Curie theorem describes equilibrium shapes, the Cahn-Hoffman results can be reconciled to the 
concept of independent chemical potentials by replacing $\pm \Delta \gamma_{1 v}$ with $\psi_{a}$ for a particle or drop on surface $a$.

PE theory will enable investigators to analyze the interrelated thermodynamics and kinetics of shape changes of bonded particles and drops by means of experimental methods and theoretical analyses already employed for isolated one-component particles. A particle of initially unstable shape, instead of following a path of maximum decrease in free energy, follows a path of successively minimum free energies of activation for atom or molecular movement. A sequence followed may strand particles in shapes that are far from the shapes prescribed by the Gibbs-Curie theorem. For particles or drops on an immobile solid, it is only necessary to replace negative gradients in $\gamma$ in rate equations for such shape changes by negative gradients in $\gamma+\lambda=\psi$. Transition state equations can be used to evaluate the importance of surface and interface steps in providing a reaction sequence that yield "engineered assemblies" of nanoscale particles like the kinetically stranded hollow nanospheres of Yen et al* (35).

In a recent review of our research on decomposition reaction kinetics (35), we describe modes of decomposition of calcite in which diffusion of $\mathrm{CO}_{2}$ from the calcite surface layers leaves $\mathrm{O}$ atoms on $\mathrm{CO}_{3}$ lattice sites. Calcite powder is often ejected from crucibles by propagation through the powder of an exothermic strain-relieving process in the destabilized layers. But in a fluidized bed reactor, $\mathrm{CaO}$ particles with cross sections as small as $1 \mu \mathrm{m}$, and potentially smaller, can be generated from similar powders at rates quantitatively predicted from rates measured at LBNL with crystals that have cross sections more than $10^{6}$ times greater (37).

While PE theory is incompatible with the mechanical model of Young and Laplace, it is consistent with equations that describe the shear rate of Newtonian liquids at a solid surface. For flow in micrometer and nanometer conduits, a key issue is whether an appreciable number of molecular layers are bonded to the solid surface more or less strongly than to a layer of molecules of the bulk liquid. (38). I am completing a paper that will guide such studies by evaluating for van der Waals liquids the dependence of $\psi$ on thickness of their nanoscale films. \# A paper by DTB and FB will illustrate the use of PE theory to predict conditions for intimate liquid phase mixing of micrometer particles of very different densities.

*et al includes Somorjai and Alivisatos.

\# This result has patent implications. A surface that attracts the first monolayer of liquid with a chemical field equal to that of the liquid itself should cause minimum thermodynamic impediment to Newtonian flow. The potential of that sub-nanoscale field can be predicted from contact angles of drops on planar solid surfaces, whether smooth or rough. 